\title{
Simulations in the Analysis of Experimental Data Measured by BM@N Drift Chambers
}

\author{
Ján Fedorišin ${ }^{1,2, \star}$ \\ ${ }^{1}$ Veksler and Baldin Laboratory of High Energy Physics, Joint Institute for Nuclear Research, \\ 141980 Dubna, Moscow region, Russia \\ ${ }^{2}$ Institute of Experimental Physics SAS, Watsonova 47, 04001 Košice, Slovakia
}

\begin{abstract}
The drift chambers (DCH's) are an important part of the tracking system of the BM@N experiment designed to study the production of baryonic matter at the Nuclotron energies.

The method of particle hit and track reconstruction in the drift chambers has been already proposed and tested on the BM@N deuteron beam data. In this study the DCH's are first locally and globally aligned, and subsequently the consistency of the track reconstruction chain is tested by two methods. The first one is based on the backward extrapolation of the DCH reconstructed deuteron beam to a position where its deflection in the BM@N magnetic field begins. The second method reconstructs the deuteron beam momentum through its deflection angle. Both methods confirm correctness of the track reconstruction algorithm.
\end{abstract}

\section{Introduction}

The BM@N (Baryonic Matter at Nuclotron) $[1,2]$ experiment is designed to study the strange matter production in collisions of heavy nuclei. BM@N is a fixed target experiment using heavy-ion beams extracted from JINR Nuclotron with the beam energies ranging from 1 to $6 \mathrm{AGeV}$. The proposed target and projectile nuclei include $\mathrm{p}, \mathrm{d}, \mathrm{C}, \mathrm{Ar}, \mathrm{Cu}, \mathrm{Xe}, \mathrm{Au}$, i.e., they cover a wide range of nuclear masses.

The drift chambers [3] constitute an important part of the BM@N tracking system. There are two identical chambers, DCH1 and DCH2, placed outside the magnetic field of the SP41 dipole magnet [4]. Each DCH consists of four segments measuring respectively $y, x, u, v$ track coordinates where $u$ and $v$ stand for the $x$ coordinate rotated by angles $\pm 45^{\circ}$ around the $z$ axis. These coordinates are used to produce charged particle tracks passing through all sixteen planes of both the drift chambers. The track reconstruction is a sophisticated process involving the detector calibration, the hits calculation as well as the track finding and fitting algorithm (all the data processing chain is thoroughly described in [5] or [6]). Furthermore, the experimental data is corrected and refined by the autocalibration procedure and the detector alignment within which, first, the DCH1 is globally aligned to the initial beam direction and position, and then the $\mathrm{DCH} 2$ is locally aligned with respect to $\mathrm{DCH} 1$. The correction procedures are tuned up on pure beam data where it is less complicated due to low multiplicities of secondary particles.

\footnotetext{
${ }^{\star}$ e-mail: fedorisin@jinr.ru
} 


\section{The method consistency checks}

Because of the complexity of the DCH track reconstruction algorithm, it is necessary to verify its accuracy and reliability. There are two basic consistency checks applied in the presented analysis, both of them try to recover the kinematic characteristics of the initial deuteron beam. Furthermore, the first method is able to reconstruct even the beam geometric parameters at an arbitrary moment of beam deflection in the magnetic field.

\subsection{Check \#1 - beam backward extrapolation}

Using Geant4 [7], the reconstructed experimental tracks are extrapolated backwards to the point where their deflection in the magnetic field begins (see Fig. 1). If everything is correct, then the following conditions hold for the coordinates: $x, y \rightarrow 0$ and the momentum components: $p_{x}, p_{y} \rightarrow 0, p_{z} \rightarrow$ $p_{\text {beam }}$.
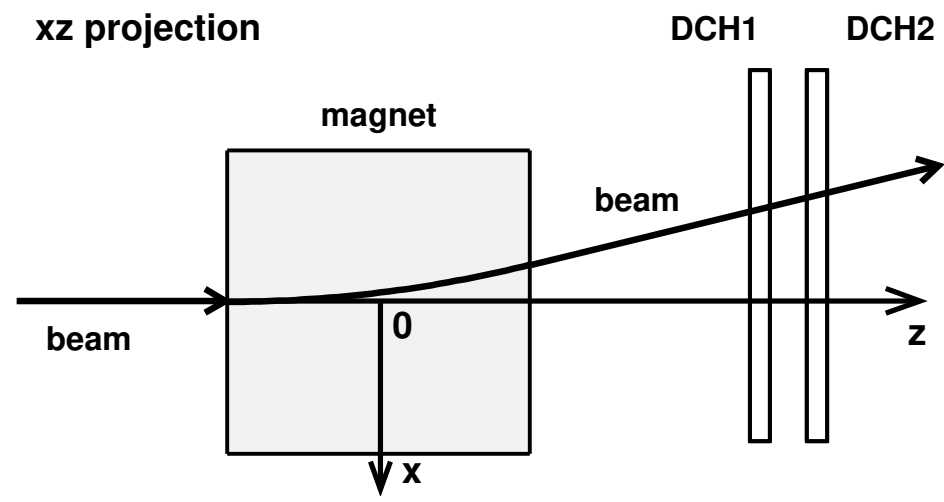

Figure 1. Layout of BM@N magnet and drift chambers

The instantiation of the results produced by the beam backward extrapolation for a typical deuteron beam run with $p_{\text {beam }}=8.68 \mathrm{GeV} / \mathrm{c}$ is shown in Figs. 2 and 3. All the kinematic and the geometric characteristics of the extrapolated beam agree within the errors with the expected values.

\subsection{Check \#2 - momentum reconstruction of the deuteron beam}

The deflection of the beam by a magnetic field in the $x z$ plane is described by the angle $\varphi_{x z}$ which depends on the magnetic field integral and the beam momentum $p$ :

$$
\varphi_{x z}=q \frac{\int B d l}{p} \quad[\mathrm{rad}] \quad \Longrightarrow \quad p=q \frac{\int B d l}{\varphi_{x z}},
$$

where $B$ stands for the $B_{y}$ component of the magnetic field and $q$ stands for the beam particle charge [8]. These relations are valid for small deflection angles when $\tan \varphi \simeq \varphi$. Fig. 4 displays the dependences of the angle $\varphi_{x z}$ and the beam momentum $p$ on the magnetic field integral. The reconstructed momentum $8.6 \pm 0.1 \mathrm{GeV} / \mathrm{c}$ is consistent with the nominal value of the deuteron momentum of $8.68 \mathrm{GeV} / \mathrm{c}$. 

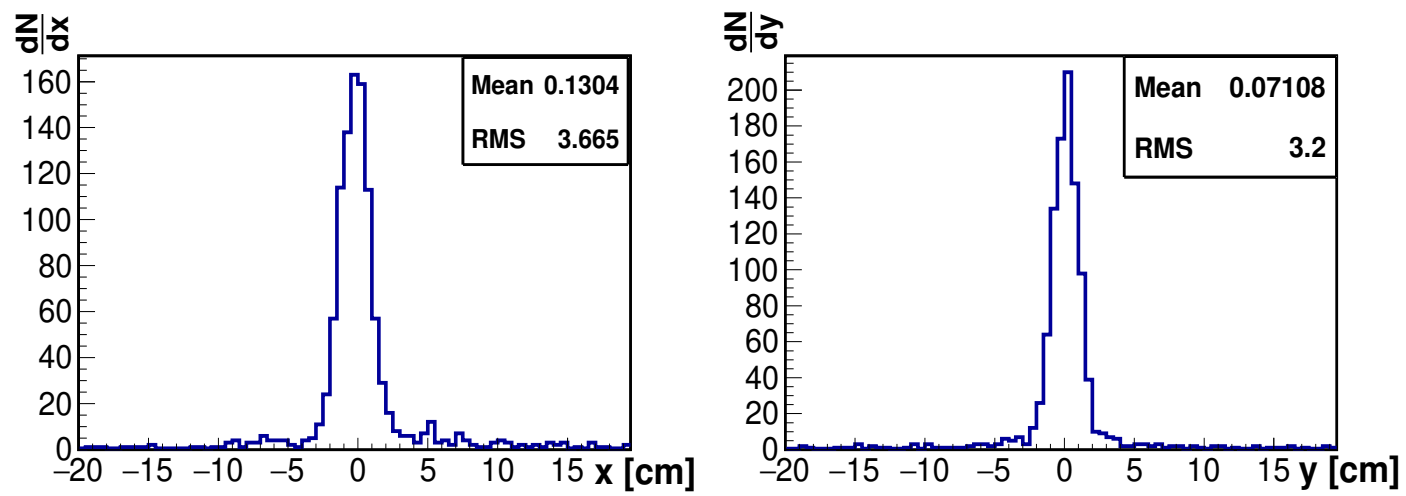

Figure 2. Restored $x$ and $y$ coordinates of the reconstructed beam at the entrance into the magnetic field
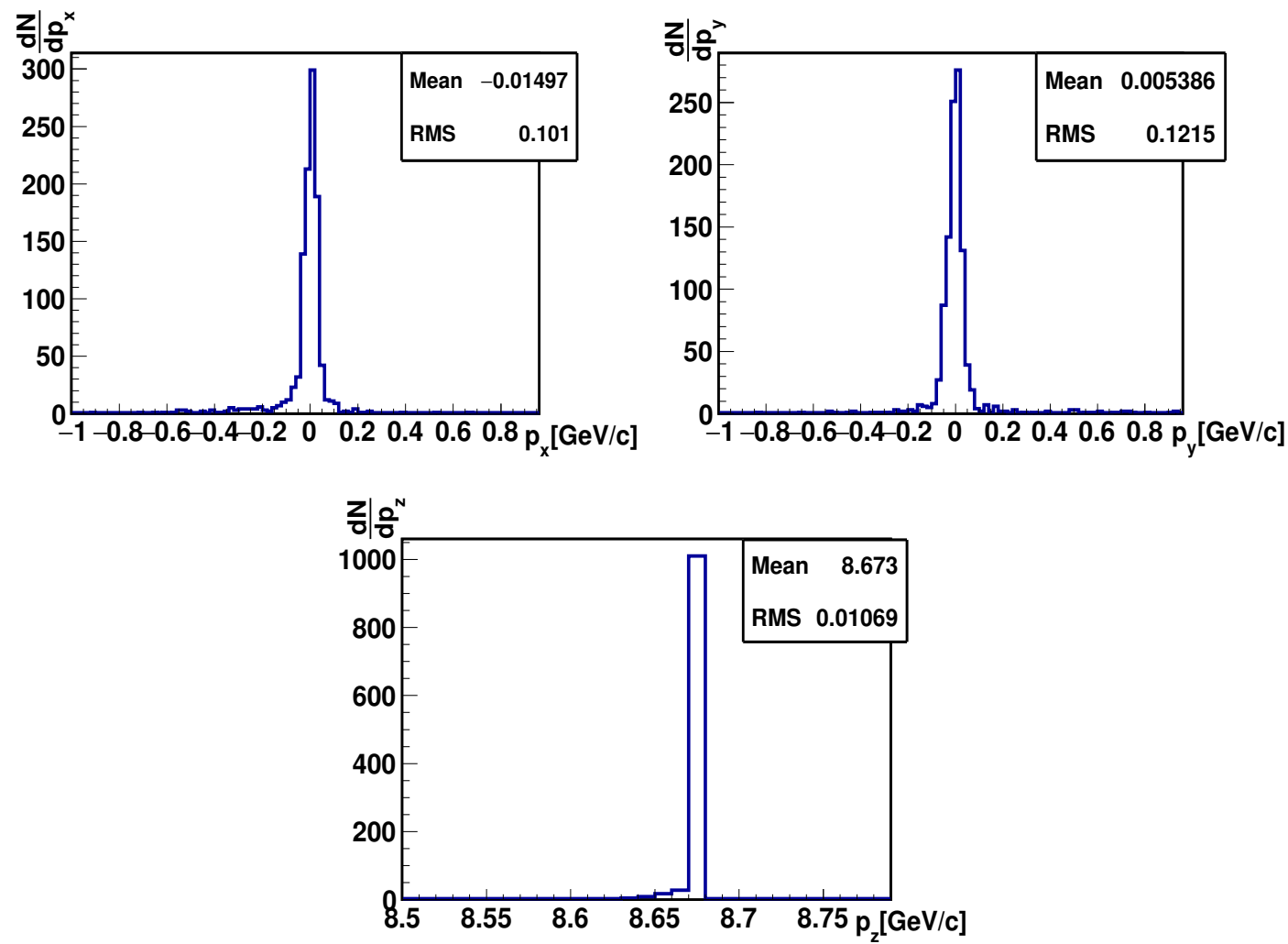

Figure 3. Restored components of the beam momentum $p_{x}, p_{y}$, and $p_{z}$, at the initial deflection point 

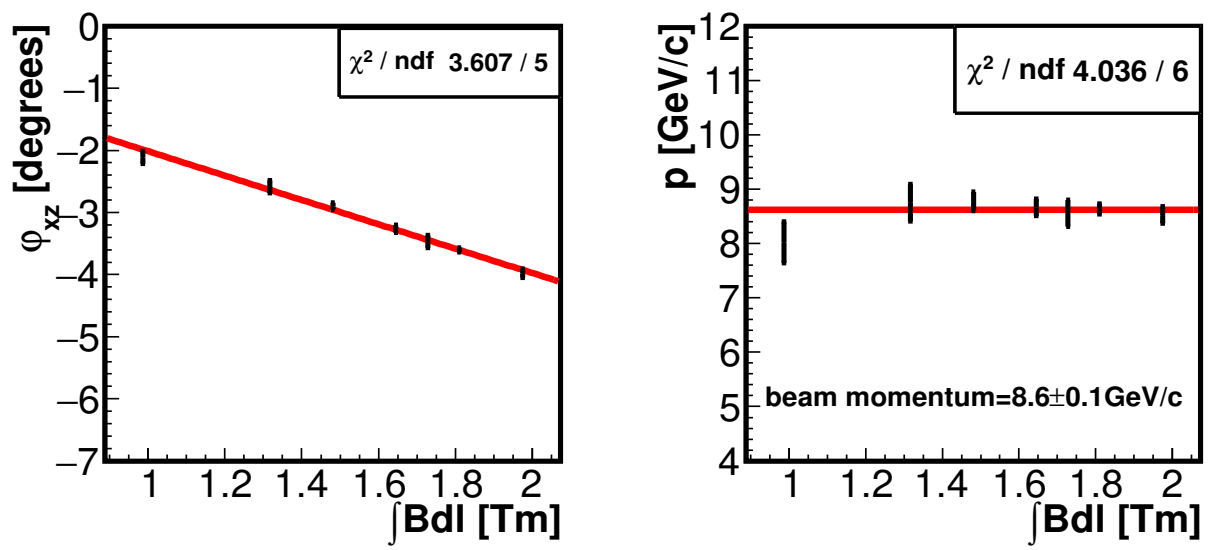

Figure 4. Deflection angle of deuteron beam in $x z$ plane (on the left) and deuteron momentum (on the right) as functions of magnetic field integral

\section{Summary}

1. A track reconstruction algorithm involving two independent consistency checks was proposed, programmed and tested to verify its accuracy and efficiency.

2. Geant 4 based track extrapolations were used in the testing procedures.

3. The two developed checks were based on the reconstruction of the initial deuteron beam kinematic and geometric characteristics - the momentum components and the $x, y$ coordinates.

4. The reconstructed values are consistent to a high degree with the corresponding initial ones.

\section{References}

[1] T.O. Ablyazimov et al., BM@N Collaboration, BM@N-Baryonic Matter at Nuclotron (Conceptual Design Report), http://nica.jinr.ru/files/BM@N/BMN_CDR.pdf (2012)

[2] M. Kapishin, Eur. Phys. J. A 52: 213 (2016)

[3] D. Béderéde et al., Nucl. Instrum. Meth. A 367, 88-91 (1995)

[4] P.G Akishin et al., physics.ins-det arXiv:1407.7096v1 (2014)

[5] J. Fedorišin, EPJ Web of Conferences 108, 02021 (2016)

[6] J. Fedorišin, EPJ Web of Conferences 138, 11005 (2017)

[7] J. Allison et al., Nucl. Instrum. Meth. A 835, 186-225 (2016)

[8] Tables of Physical \& Chemical Constants (16th edition 1995). Kaye \& Laby Online. Version 1.0 (2005) http://www.kayelaby.npl.co.uk 